\title{
An Investigation Into the Effects of Magnesium Sulfate on the Complications of Succinylcholine Administration in Nulliparous Women Undergoing Elective Cesarean Section: A Double-Blind Clinical Trial
}

\author{
Davoud Aghamohamadi ${ }^{1 \mathbb{D}}$, Mehdi Khanbabayi Gol ${ }^{2 * \mathbb{D}}$
}

\begin{abstract}
Objectives: Controlling the complications of anesthetics (e.g., succinylcholine) seems necessary since they are greater for nulliparous women who undergo elective cesarean section (C-section). The present study aimed to investigate the effects of magnesium sulfate on the complications of succinylcholine administration in nulliparous women undergoing elective C-section.

Materials and Methods: This double-blind clinical trial was conducted on 60 nulliparous women during 2012-2013. The women were randomly assigned to A and B groups. Before inducing the rapid anesthesia with succinylcholine, patients in the intervention group received $30 \mathrm{mg} / \mathrm{kg}$ of magnesium sulfate within 10 minutes and then the hemodynamic status and the side effects of succinylcholine were recorded in a special form. Statistical tests were performed using repeated-measures ANOVA, chi-square test, and one-way ANOVA tests and the level of significance was determined to be $P<0.05$.

Results: There was no significant difference between the two groups in terms of demographic data $(P>0.059)$. However, the results indicated that there was a significant difference between the 2 groups regarding the myoglobin level $(P=0.010)$. Contrarily, the results showed that most patients in the intervention $(n=23)$ and control $(n=15)$ groups experienced no or mild and severe fasciculation, respectively $(P<0.001)$.

Conclusions: In general, magnesium sulfate can greatly control and reduce the complications of succinylcholine administration, including fasciculation.

Keywords: Succinylcholine, Fasciculation, Magnesium sulfate
\end{abstract}

\section{Introduction}

Statistics show that the number of cesarean sections (C-sections) is increasing worldwide (1). According to many health professionals and authorities, this increase can be higher in Iran because different C-sections are performed without scientific indication and by the mother's choice $(2,3)$.

General anesthesia should be employed in many cases such as bleeding, coagulopathies, neonatal lifethreatening disorders, or for those who do not accept or tolerate local anesthetic induction. In addition, pregnant women undergoing a C-section are among the most sensitive groups that should be intubated based on rapid sequencing, and there is no choice but to prescribe succinylcholine for such subjects $(4,5)$. Due to the specificity of succinylcholine in developing a rapid pattern in the anesthetic induction sequence, this drug is widely used for patients with impaired respiratory tracts, especially pregnant women undergoing a C-section. The mechanism of the effect of succinylcholine is similar to that of acetylcholine. Accordingly, succinylcholine can effectively cause a continuous non-polar state in a competitive process with acetylcholine in the pre-synaptic part of the nerve terminal. Unlike acetylcholine, this drug is hydrolyzed very slowly and thus the membrane remains abnormal for a while, leading to the long-term rest of the ion channel that accompanies the extracellular secretion of potassium (6).

Similarly, succinylcholine is the most common drug that is utilized as the intubation facilitator, especially in rapid sequencing. However, because of its side effects, researchers are seeking to find alternative drugs. Such side effects of succinylcholine include cardiovascular conditions (i.e., sinus bradycardia, nodal rhythms, and ventricular dysrhythmias), hyperkalemia, increased intraocular pressure, increased intra-abdominal pressure, increased intracranial pressure, fibromyalgia, muster muscle spasm, as well as the risk of malignant hyperthermia, rhabdomyolysis, and prolonged muscle relaxation time (7). Since succinylcholine is the most prevalently used relaxants for rapid induction in pregnant women, it is very essential to identify its complications and apply measures 
to reduce these problems (8). Therefore, various drugs were tested to decrease the side effects of succinylcholine. For example, magnesium sulfate is known to be a calcium channel blocker, which is an antagonist to N-methyl-Daspartate receptors (9) and is widely used in the C-section since it has the least side effects on mother and fetus (10). In this regard, magnesium sulfate is considered as one of these drugs. It is believed that magnesium sulfate can reduce fasciculation and prevent acetylcholine secretion because magnesium affects the neuromuscular site and prevents acetylcholine secretion in competition with calcium (11).

Considering the above-mentioned explanations, preventing the unwanted side effects of succinylcholine in women candidates for elective C-section is an inevitable necessity that requires further studies. On the other hand, nulliparous women are more sensitive than other women to the choice of anesthesia method and complications of anesthetics. The complications of anesthetics, including succinylcholine, are greater in such women as well (12) thus there is a need for prescribing a drug which has the least side effects on the mother and the fetus. Hence, the current study sought to evaluate the effects of magnesium sulfate on the complications of succinylcholine in nulliparous women who undergo an elective C-section.

\section{Materials and Methods}

The present study was a double-blind clinical trial with a control group (identifier: IRCT201107221772N8; https://www.irct.ir/trial/133) and was performed on 60 nulliparous women who attended Al-Zahra hospital of Tabriz for delivery from 20 September 2012 to 20 September 2013. Considering $\alpha=0.05, \beta=0.2$, and $d=1.4$ and based on the fasciculation variable, the sample size was determined to be 30 for each group (13). The women were selected using a convenience sampling method and then were randomly divided into the control (A) and intervention (B) groups.

The only inclusion criterion included the willingness for participation in the study and the exclusion criteria were as follows:

- Muscle aches before anesthesia;

- Affliction with kidney or metabolic diseases;

- Myopathy or muscle-nerve disorders;

- Symptomatic urinary tract infection and hematuria'

- Trauma over the last two months or burning over the last 2 years;

- The history of muscular dystrophy in the patient or their first-degree relatives;

- The prohibition of succinylcholine administration (i.e., the history of malignant hyperthermia or cholinesterase deficiency;

- Preeclampsia;

- Sensitivity to magnesium sulfate and blood transfusion during the surgery.

In the operating room, there were two types of $1000-\mathrm{cc}$ infusion sets containing the solution of group B and the placebo solution that were prepared by an anesthesia technician who was aware of the research procedure. One of the infusion sets included the normal saline and the other one contained $30 \mathrm{mg} / \mathrm{kg}$ of magnesium sulfate and normal saline (13). Patients of group B received magnesium sulfate. After placing on the bed of the operating room, the subjects were examined and monitored in terms of blood pressure, heart rate, and oxygen saturation (SpO2). Next, the relevant solutions were administered to the subjects in 10 minutes, followed by inducing the anesthesia using propofol $(1.5 \mathrm{mg} / \mathrm{kg})$ and succinylcholine $(1.5 \mathrm{mg} /$ $\mathrm{kg}$ ). After 20 seconds, laryngoscopy and endotracheal intubations were performed for each subject. During all stages of induction, the Sellick maneuver was continued and the subjects' respiration was supported by the anesthetic machine after blowing the cuff of the endotracheal tube and fixing its location. In addition, anesthesia was continued with $0.8 \%$ isoflurane, $50 \%$ oxygen, and $\mathrm{N}_{2} \mathrm{O}$. Further, atracurium was then added to the drugs in the early minutes of anesthesia after the patient's respiratory recovery. Next, the vital signs were measured before the administration and the induction of anesthesia at minutes 1,3 , and 5 after intubation and then every 15 minutes during the surgery. These measures were performed for all subjects.

Likewise, fasciculation was measured within the first 30 seconds after succinylcholine administration and was categorized as no or mild fasciculation (0), fasciculation of the eyes, face, neck, or the fingers without the whole organ movement (Fine), the clear twitching of muscles in more than one place with the entire organ movement (Moderate), and continuous fasciculation (Severe).

Moreover, the blood samples were obtained from the subjects in order to measure the level of magnesium, potassium, and creatine phosphokinase before prescribing the drug and 20 minutes after inducting the anesthesia. In addition, the urine samples were collected using a urine catheter and then were transferred to the laboratory for measuring myoglobin 20 minutes after anesthesia induction.

The hemodynamic variables were also measured every 15 minutes during the operation and in the recovery room and recorded in a special form prepared for this purpose. Arterial SpO2 and the number of breaths were measured to evaluate the respiratory status of the subjects. The severity of the muscle pain was also estimated based on the Numerical Pain Rating Scale 24 hours after the surgery. On this scale, 0 represents no pain while 10 denotes severe pain. Moreover, the following parameters were measured and recorded based on the modified Aldrete's scoring system:

- The duration of anesthesia from the induction of anesthesia to the anesthetic discontinuation;

- The duration of surgery from cutting to dressing;

- The duration of the tracheal tube extraction from 
anesthetic discontinuation to extubation;

- The duration of recovery from admission to the recovery unit to discharge. According to this system, patients who gain a score of 7 can be safely discharged from the recovery unit.

Data were analyzed by SPSS20 software using repeatedmeasures ANOVA, chi-square, and one-way ANOVA tests and the significance level was considered as $P<0.05$.

Additionally, the ethical considerations were completely observed similar to those of other studies $(14-17,18)$. The subjects and their first-degree relatives were briefed on the research objectives and procedures. Then, an informed consent form was obtained from all subjects and they were assured of discontinuing the study at any stage.

\section{Results}

The present study was conducted with two groups (A and $\mathrm{B}$ ) and none of the subjects left the study (subject's mortality rate was equal to zero).

According to Table 1, none of the variables of age, height, weight, and body mass index were statistically different between the two groups.
Contrarily, a significant difference was found between the two groups after the evaluation of the myoglobin level. $(P=0.010)$, indicating the positive result in myoglobin in 12 and 5 cases in groups A (40\%) and B (16.66\%), respectively. The evaluation of hemodynamic parameters during the surgery and recovery in both groups also showed a significant difference in all parts (Table 2 ).

The results further demonstrated a significant difference between the two groups regarding fasciculation 30 seconds after succinylcholine administration, representing that most women in groups $B(n=23)$ and $A$ $(n=15)$ experienced no or mild fasciculation and severe fasciculation, respectively $(P<0.001)$, the details of which are provided in Table 2.

In addition, the results indicated that the mean severity of pain in group $B(1.75 \pm 1.20)$ was significantly lower $(P<0.001)$ than that of group A $(6.20 \pm 2.15)$.

As shown in Table 3, there was also a significant difference in the serum levels of magnesium $(P<0.001)$ and potassium $(P=0.001)$ whereas such a difference was not observed between them in terms of creatine phosphokinase $(P<0.640)$.

Table 1. Demographic Characteristics of the Subjects

\begin{tabular}{|c|c|c|c|}
\hline \multirow{2}{*}{ Variable } & \multicolumn{2}{|c|}{ Group $(N=60)$} & \multirow{2}{*}{$P$ Value } \\
\hline & Intervention $(n=30)$ & Witness $(n=30)$ & \\
\hline Age (mean $\pm S D)$ & $30.34 \pm 4.55$ & $29.81 \pm 5.61$ & 0.650 \\
\hline Weight $(\mathrm{kg})$ (mean \pm SD) & $80.30 \pm 1.48$ & $82.01 \pm 5.11$ & 0.461 \\
\hline Height $(\mathrm{cm})($ mean $\pm \mathrm{SD})$ & $167.55 \pm 6.60$ & $165.25 \pm 5.90$ & 0.060 \\
\hline $\mathrm{BMI}\left(\mathrm{kg} / \mathrm{m}^{2}\right)($ mean $\pm \mathrm{SD})$ & $29.41 \pm 3.50$ & $31.28 \pm 3.21$ & 0.135 \\
\hline
\end{tabular}

Abbreviations: SD, standard deviation; BMI, body mass index.

Table 2. Comparison of Subjects in the Intervention and Control Groups in Terms of Hemodynamic Variables

\begin{tabular}{|c|c|c|c|}
\hline \multirow{2}{*}{ The Studied Variable } & \multicolumn{2}{|c|}{ Groups (N=60) } & \multirow{2}{*}{$P$ Value } \\
\hline & Intervention $(\mathrm{n}=30)$ & Control $(n=30)$ & \\
\hline HR in the first minute after intubation & $70.75 \pm 7.69$ & $77.20 \pm 8.54$ & 0.011 \\
\hline HR in the third minute after intubation & $65.23 \pm 6.11$ & $72.25 \pm 7.05$ & 0.020 \\
\hline HR in the Fifth minute after intubation & $68.30 \pm 7.35$ & $75.35 \pm 7.50$ & 0.015 \\
\hline HR during surgery & $68.25 \pm 7.45$ & $75.25 \pm 7.80$ & 0.015 \\
\hline $\mathrm{HR}$ in PACU & $82.5 \pm 7.89$ & $89.20 \pm 8.91$ & 0.021 \\
\hline Systolic BP in the first minute after intubation & $115.12 \pm 10.53$ & $125.50 \pm 9.28$ & 0.030 \\
\hline Systolic BP in the third minute after intubation & $111.25 \pm 11.33$ & $120.60 \pm 11.55$ & 0.039 \\
\hline Systolic BP in the Fifth minute after intubation & $108.50 \pm 11.11$ & $115.12 \pm 10.53$ & 0.018 \\
\hline Systolic BP during surgery & $110.25 \pm 11.35$ & $125.70 \pm 11.70$ & 0.020 \\
\hline Systolic BP in PACU & $120.00 \pm 8.97$ & $129.50 \pm 10.78$ & 0.036 \\
\hline Diastolic BP in the first minute after intubation & $62.61 \pm 2.37$ & $70.92 \pm 2.11$ & 0.017 \\
\hline Diastolic BP in the third minute after intubation & $60.25 \pm 2.50$ & $65.55 \pm 2.50$ & 0.018 \\
\hline Diastolic BP in the Fifth minute after intubation & $53.33 \pm 2.15$ & $62.11 \pm 2.30$ & 0.019 \\
\hline Diastolic BP during surgery & $51.61 \pm 2.15$ & $60.29 \pm 2.3497$ & 0.030 \\
\hline Diastolic BP in PACU & $55.54 \pm 3.27$ & $62.68 \pm 2.09$ & 0.011 \\
\hline $\mathrm{SpO} 2$ in the first minute after intubation & $98.62 \pm 0.88$ & $95.72 \pm 4.08$ & 0.019 \\
\hline $\mathrm{SpO} 2$ in the third minute after intubation & $98.25 \pm 0.20$ & $96.20 \pm 2.88$ & 0.025 \\
\hline $\mathrm{SpO} 2$ in the Fifth minute after intubation & $98.33 \pm 0.42$ & $96.62 \pm 2.11$ & 0.023 \\
\hline $\mathrm{SpO} 2$ during surgery & $98.21 \pm 0.11$ & $96.15 \pm 1.40$ & 0.015 \\
\hline $\mathrm{SpO} 2$ in PACU & $98.20 \pm 0.62$ & $96.32 \pm 0.72$ & 0.019 \\
\hline
\end{tabular}

Abbreviations: BP, Blood pressure; PACU, post-anesthesia care unit; HR: Heart rate; SpO2, oxygen saturation. 
Table 3. Evaluation of Fasciculation in Subjects 30 Seconds After Succinylcholine Administration

\begin{tabular}{|c|c|c|c|}
\hline \multirow{2}{*}{ Severity of Fasciculation } & \multicolumn{2}{|c|}{ Groups (N=60) } & \multirow{2}{*}{$P$ Value } \\
\hline & Intervention $(n=30)$ & Control $(n=30)$ & \\
\hline 0, No. (\%) & $18(60)$ & $3(10)$ & \multirow{4}{*}{$<0.001$} \\
\hline Fine, No. (\%) & $5(16.66)$ & $3(10)$ & \\
\hline Moderate, No. (\%) & $4(13.34)$ & $9(30)$ & \\
\hline Severe, No. (\%) & $3(10)$ & $15(50)$ & \\
\hline
\end{tabular}

Table 4. Pre- and Post-intervention Serum Levels of Magnesium, Potassium, and CPK in the Studied Groups

\begin{tabular}{|c|c|c|c|c|}
\hline \multirow{2}{*}{ Variable } & \multirow{2}{*}{ Time } & \multicolumn{2}{|c|}{ Groups $(\mathrm{N}=60)$} & \multirow{2}{*}{$P$ Value } \\
\hline & & Intervention $(n=30)$ & Control $(n=30)$ & \\
\hline \multirow{3}{*}{ Magnesium, mean $\pm S D$} & Before intervention & $1.30 \pm 0.61$ & $2.25 \pm 0.61$ & \\
\hline & After the intervention & $2.80 \pm 0.58$ & $2.45 \pm 0.55$ & $<0.001$ \\
\hline & $P$ value & $<0.001$ & 0.140 & \\
\hline \multirow{3}{*}{ Serum potassium, mean \pm SD } & Before intervention & $3.91 \pm 0.33$ & $4.11 \pm 0.25$ & \\
\hline & After the intervention & $4.43 \pm 0.20$ & $4.20 \pm 0.31$ & 0.001 \\
\hline & $P$ value & 0.001 & 0.381 & \\
\hline \multirow{3}{*}{$\mathrm{CPK}$, mean $\pm \mathrm{SD}$} & Before intervention & $119.85 \pm 40.63$ & $115.65 \pm 41.28$ & 0.640 \\
\hline & After the intervention & $125.65 \pm 41.20$ & $109.30 \pm 39.50$ & \\
\hline & $P$ value & 0.190 & 0.118 & \\
\hline
\end{tabular}

Abbreviations: SD, standard deviation; CPK, creatine phosphokinase.

\section{Discussion}

The current study attempted to investigate the role of magnesium sulfate in the complications of succinylcholine administration among nulliparous women undergoing elective cesarean section (C-section). As a non-polar muscle relaxant with agonistic properties, succinylcholine causes skeletal muscles to quickly relax and return to the normal state after a few minutes. However, this drug leads to many combinations, and various substances such as magnesium sulfate are used to counteract its complications $(19,20)$.

Magnesium blockade affects the site of neuromuscular involvement, as well as the inhibition of acetylcholine release. In addition, it can enhance the effect of nondepolarizing muscle relaxants, therefore, magnesium sulfate injection is confirmed to have positive effects on the reduction of complications from succinylcholine at times by using various surgical procedures (21).

The study groups failed to differ in terms of demographic characteristics. Therefore, it can be stated that the results were directly caused by interventional mechanisms. Based on the results of the study, the hemodynamic status in patients treated with magnesium sulfate was significantly more stable as compared to those in group A. Similar to this study, several previous studies reported the hemodynamic stability of patients following the administration of magnesium sulfate $(10,22)$.

The results showed that there was a significant increase in the serum level of magnesium in group B. The serum level of potassium represented an increase in both groups but there was no significant difference between them in this regard. In addition, potassium reached no dangerous levels for arrhythmia in both groups. The administration of magnesium sulfate also reduced myoglobinuria effectively. On the other hand, the severity of fasciculation and the pain score were significantly lower in group B 24 hours after the surgery.

Many studies approved positive and beneficial effects of magnesium sulfate on the facilitation of intubation and laryngoscopy, along with hemodynamic stability during laryngoscopy (23-25), which is in line with the findings of the current study.

In the present study, the preventive administration of magnesium sulfate in group B reduced the severity of fasciculation following the injection of succinylcholine. Further, other studies confirmed the findings of the present study regarding the positive and beneficial effects of the prophylactic administration of magnesium sulfate on fasciculation control (26-28). It seems that magnesium sulfate prevents severe fasciculation reactions by inhibiting acetylcholine secretion after the injection of succinylcholine. In addition, magnesium sulfate reduces postoperative muscle aches in patients who receive succinylcholine.

Hashemi et al studied the effects of different doses of magnesium sulfate on the reduction of complications caused by the injection of succinylcholine and reported that higher doses of magnesium sulfate can further control the subsequent complications of succinylcholine, including fasciculation (29). However, it seems that the increased dosage of magnesium sulfate can cause adverse effects for the patients by affecting magnesium and potassium serum levels. Similarly, the negative effects of magnesium sulfate on the embryo in pregnant women justify the limited use of its higher doses since both the embryo and the mother are of high priority in elective C-sections not merely 
the mother and maternal complications. This point was mostly ignored in the above-mentioned studies.

Limitations of the Study

The main research limitation was the focus on the elective $\mathrm{C}$-section and inattention to emergency C-sections whereas succinylcholine is more widely used in emergency procedures. Hence, more studies are recommended in this regard by focusing on emergency C-sections. Further, the lack of attention to maternal and neonatal complications during the postpartum period is considered as another limitation of the study, which should be highlighted in future studies.

\section{Conclusions}

Overall, magnesium sulfate can greatly control and reduce the complications of succinylcholine administration, including fasciculation. Thus, its preventive administration can stabilize the hemodynamic status and properly control postoperative pain.

\section{Conflict of Interests}

Authors declare that they have no conflict of interests.

\section{Ethical Issues}

This article has been registered by the Ethics Committee (Ethic No. 5/4/323) of Tabriz University of Medical Sciences.

\section{Financial Support}

The researchers thank Tabriz University of Medical Sciences for financial support.

\section{References}

1. Lobel M, DeLuca RS. Psychosocial sequelae of cesarean delivery: review and analysis of their causes and implications. Soc Sci Med. 2007;64(11):2272-2284. doi:10.1016/j.socscimed.2007.02.028

2. Irani M, Kordi M, Lotfalizadeh M. Methods of Assessing the Labor Progress: A Review Study. The Iranian Journal of Obstetrics, Gynecology and Infertility. 2019;21(11):80-90. doi:10.22038/ijogi.2019.12330

3. Amiri Farahani L, Abbasi Shavazi MJ. Caesarean section change trends in Iran and some demographic factors associated with them in the past three decades. Journal of Fasa University of Medical Sciences. 2012;2(3):127-134. [Persian].

4. Parmar S, Vyas A, Sheikh A. Usefulness of propofol to prevent succinylcholine induced fasciculations and myalgia, a comparison with thiopentone sodium as an induction agent. Int J Med Sci Public Health. 2013;2(2):339-343. doi:10.5455/ijmsph.2013.2.353-357

5. Roy S, Mrunalini K, Venkateshwarlu G, Sowmya Sri A. Comparative study on the effects of pretreatment with magnesium sulphate and propofol induction on serum creatine phosphokinase and urinary myoglobin levels associated with the use of succinylcholine. J Evol Med Dent Sci. 2015;4(55):9568-9580. doi:10.14260/jemds/2015/1383
6. Piotrowski AJ, Fendler WM. Hyperkalemia and cardiac arrest following succinylcholine administration in a 16-year-old boy with acute nonlymphoblastic leukemia and sepsis. Pediatr Crit Care Med. 2007;8(2):183-185. doi:10.1097/01.pcc.0000257103.96579.b2

7. Miller RD, Eriksson LI, Fleisher LA, Wiener-Kronish JP, Cohen NH, Young WL. Miller's Anesthesia E-Book. Elsevier Health Sciences; 2014.

8. O'Connor RE, McGinnis-Hainsworth D, Megargel R. 3: Prospective Evaluation of Etomidate With and Without the Addition of Succinylcholine for Rapid Sequence Intubation of Head Injured Patients in the Out-of-Hospital Setting. Ann Emerg Med. 2007;50(3):S2. doi:10.1016/j. annemergmed.2007.06.031

9. Begum R, Begum A, Bullough CH, Johanson RB. Reducing maternal mortality from eclampsia, using magnesium sulphate. Eur J Obstet Gynecol Reprod Biol. 2000;92(2):223224. doi:10.1016/s0301-2115(99)00274-2

10. Alipour M, Sharifian A, Dastkhosh A. Effects of magnesium sulfate on prevention of shivering during spinal anesthesia in cesarean section. The Iranian Journal of Obstetrics, Gynecology and Infertility. 2013;16(79):1-9. doi:10.22038/ ijogi.2013.2166

11. Kumar M, Talwar N, Goyal R, Shukla U, Sethi A. Effect of magnesium sulfate with propofol induction of anesthesia on succinylcholine-induced fasciculations and myalgia. J Anaesthesiol Clin Pharmacol. 2012;28(1):81-85. doi:10.4103/0970-9185.92451

12. Hosseini Valami M, Hosseini Jahromi A, Mahmoodi T, Barikani A. Comparing the Effects of Intraperitoneal Injection of Bupivacaine, Morphine, and Dexamethasone on Pain after Elective Caesarean Section under General Anesthesia. Journal of Mazandaran University of Medical Sciences. 2017;27(147):139-149.

13. Aghamohammadi D, Farzin $H$, Khanbabayi Gol $M$, Fooladi S. The effects of intravenous magnesium sulfate on hemodynamic status and pain control in patients after laparotomy surgery: A double blind clinical trial. Journal of Anesthesiology and Pain. 2018;9(1):66-73. [Persian].

14. Movassaghi R, Peirovifar A, Aghamohammadi D, Mohammadipour Anvari H, Golzari SE, Kourehpaz Z. Premedication With Single Dose of Acetazolamide for the Control of Referral Shoulder Pain After Laparoscopic Cholecystectomy. Anesth Pain Med. 2015;5(6):e29366. doi:10.5812/aapm.29366

15. Bakhshaei MH, Manuchehrian N, Khoshraftar E, Mohammadipour Anvari H, Sanatkarfar M. Analgesic effects of intrathecal sufentanil added to lidocaine 5\% in elective cesarean section. Acta Med Iran. 2010;48(6):380384.

16. Fakhari S, Bavil FM, Bilehjani E, Abolhasani S, Mirinazhad M, Naghipour B. Prophylactic furosemide infusion decreasing early major postoperative renal dysfunction in on-pump adult cardiac surgery: a randomized clinical trial. Res Rep Urol. 2017;9:5-13. doi:10.2147/rru.s126134

17. Atashkhoei S, Fakhari S, Pourfathi H, Bilehjani E, Garabaghi PM, Asiaei A. Effect of oxytocin infusion on reducing the blood loss during abdominal myomectomy: a double-blind randomised controlled trial. BJOG. 2017;124(2):292-298. doi:10.1111/1471-0528.14416

18. Zomorrodi A, Mohammadipoor Anvari H, Kakaei F, 
Solymanzadeh F, Khanlari E, Bagheri A. Bolus Injection Versus Infusion of Furosemide in Kidney Transplantation: A Randomized Clinical Trial. Urol J. 2017;14(2):3013-3017. doi:10.22037/uj.v14i2.3787

19. Abdollahi H, Tirgar-Fakheri K, Eskandari M, et al. The effects of succinylcholine and remifentanil on intubating conditions in elective cesarean section. Journal of Isfahan Medical School. 2017;35(451):1432-1437. [Persian].

20. Raisi M, Abutalebi Nasr Abad S. Conducted to evaluate the efficacy of Atracurium or lidocaine alone or together on succinylcholine induced fasciculation in general anesthesia surgeries. Scientific Journal Management System. 2012;2(2):1-15. [Persian].

21. Sakuraba S, Serita R, Kosugi S, Eriksson LI, Lindahl SG, Takeda J. Pretreatment with magnesium sulphate is associated with less succinylcholine-induced fasciculation and subsequent tracheal intubation-induced hemodynamic changes than precurarization with vecuronium during rapid sequence induction. Acta Anaesthesiol Belg. 2006;57(3):253-257.

22. Heydari A, Kariman N, Naeje Z, Ahmadi F. Effect of Topical Application of Magnesium Sulfate on the Intensity of Labor Pain: Double Blind Clinical Trial. The Iranian Journal of Obstetrics, Gynecology and Infertility. 2018;21(5):58-65. doi:10.22038/ijogi.2018.11385

23. Hirmanpour A, Safavi MR, Talakoub R, Honarmand A, Ghasri M, Chitsaz N. The Effect Comparison of Two Injections Doses of Magnesium Sulfate on Hemodynamic Changes Caused by Laryngoscopy and Endotracheal Intubation in Caesarean Patients with General Anesthesia. Arch Anesthesiol Crit Care. 2017;3(1):273-277.
24. Soltani HA, Hashemi SJ, Montazeri K, Dehghani A, Nematbakhsh M. The role of magnesium sulfate in tracheal intubation without muscle relaxation in patients undergoing ophthalmic surgery. J Res Med Sci. 2016;21:96. doi:10.4103/1735-1995.193168

25. Do SH. Magnesium: a versatile drug for anesthesiologists. Korean J Anesthesiol. 2013;65(1):4-8. doi:10.4097/ kjae.2013.65.1.4

26. Raman A, Singh RK, Sarwer F, et al. Effect of pretreatment with magnesium sulphate on succinylcholineinduced fasciculations and myalgia. J Evid Based Med Healthc. 2016;3(102):5600-5606. doi:10.18410/jebmh/2016/1159

27. Yassin HM, Abdel Moneim AT, Mostafa Bayoumy AS, Bayoumy HM, Taher SG. The Influence of Two Different Doses of Magnesium Sulfate on Intraocular Pressure Variations after Injection of Succinylcholine and Endotracheal Intubation: A Prospective, Randomized, Parallel Three-Arm, Double-blind, Placebo-controlled Clinical Trial. Anesth Essays Res. 2017;11(4):969-975. doi:10.4103/aer.AER_35_17

28. Ahsan B, Rahimi E, Moradi A, Rashadmanesh N. The effects of magnesium sulphate on succinylcholine-induced fasciculation during induction of general anaesthesia. J Pak Med Assoc. 2014;64(10):1151-1153.

29. Hashemi SJ, Hirmandpour A, Namazi M, Hoseini E. The Effect of Two Different Prophylactic Doses of Magnesium Sulfate on Reduction of Succinylcholine Complications in Elective Cesarean Section under General Anesthesia in Comparison with Control Group. Journal of Isfahan Medical School. 2018;36(488):800-806. doi:10.22122/jims. v36i488.9628

(C) 2019 The Author (s); This is an open-access article distributed under the terms of the Creative Commons Attribution License (http://creativecommons.org/licenses/by/4.0), which permits unrestricted use, distribution, and reproduction in any medium, provided the original work is properly cited. 